\title{
Fast Detection of Infrared Laser Pulses on Planar Small Area GaAs/AlGaAs Heterojunction
}

E. Širmulis*, V. Kazlauskaitė, J. Gradauskas, A. SuŽIEdĖlis AND S. AŠMONTAS

Semiconductor Physics Institute

A. Goštauto 11, LT-01108 Vilnius, Lithuania

\begin{abstract}
We report the first experimental observation of fast photovoltage in small area GaAs/AlGaAs heterojunction planar detector induced by nanosecond $\mathrm{CO}_{2}$ laser pulses. This device revealed itself as a sensitive detector of millimeter waves and as a fast IR sensor operating at room temperature. Taking into account fast response of the detector and close to linear voltage-power dependence, the results are interpreted by photoemission of hot carriers over the potential barrier of the heterojunction.
\end{abstract}

PACS numbers: 72.40.+w, 73.40.Kp

\section{Introduction}

Detectors fabricated from GaAs/AlGaAs heterojunctions were successfully used to detect electromagnetic radiation at microwaves [1]. Previous experiments showed that similar heterojunctions can be used to detect IR laser radiation as well [2]. In this report we present an experimental investigation of photovoltage in small area GaAs/AlGaAs planar detectors induced by pulsed microwaves and $\mathrm{CO}_{2}$ laser.

\section{Experimental details}

GaAs/AlGaAs heterostructures for the detector fabrication were grown onto GaAs substrate by MBE technique. The order of layers in the device and the conduction band energy diagram are depicted in Fig. 1. The samples were fabricated using photolithography technique with metallic contacts set onto highly doped regions on both sides of the heterostructure.

*corresponding author; e-mail: sirmulis@delfi.lt 


\begin{tabular}{|c|}
\hline$n^{+}-\mathrm{GaAs}, N_{d}=4 \times 10^{18} \mathrm{~cm}^{-3}, h=10 \mathrm{~nm}$ \\
\hline$n^{+}-\mathrm{Al}_{0.3} \mathrm{Ga}_{0.7} \mathrm{As}, N_{d}=3 \times 10^{18} \mathrm{~cm}^{-3}, h=40 \mathrm{~nm}$ \\
\hline$n-\mathrm{Al}_{0.3} \mathrm{Ga}_{0.7} \mathrm{As}, N_{d}=10^{16} \mathrm{~cm}^{-3}, h=300 \mathrm{~nm}$ \\
\hline$n-\mathrm{GaAs}, N_{d}=10^{16} \mathrm{~cm}^{-3}, h=300 \mathrm{~nm}$ \\
\hline$n^{+}-\mathrm{GaAs}, N_{d}=3 \times 10^{18} \mathrm{~cm}^{-3}, h=300 \mathrm{~nm}$ \\
\hline$i-\mathrm{Al}_{0.3} \mathrm{Ga}_{0.7} \mathrm{As}, h=10 \mathrm{~nm}$ \\
\hline$i-\mathrm{GaAs}, h=4000 \mathrm{~nm}$ \\
\hline
\end{tabular}

Fig. 1. Schematic view of the heterostructure cross section (a) and conduction band diagram (b).

For the measurements in the microwave range the klystron $(26 \div 37.5 \mathrm{GHz})$ and travelling-wave tube $(75 \div 110 \mathrm{GHz}$ and $110 \div 178 \mathrm{GHz})$ were used. Excitation at wavelength $9.2 \mu \mathrm{m}$ was obtained from pulsed $\mathrm{CO}_{2}$ laser. The laser pulse shape and peak intensity were controlled using liquid nitrogen $\left(\mathrm{LN}_{2}\right)$ cooled $\mathrm{Ge}\langle\mathrm{Au}\rangle$ photodetector. All measurements were performed at room temperature.

\section{Results and discussion}

Due to low energetic barrier $(\approx 0.2 \div 0.3 \mathrm{eV})$ of GaAs/AlGaAs heterojunction, the differential electrical resistance of the detector was low $(600 \div 800 \Omega)$, which guaranteed fast and bias-less detection of the microwave signals. Frequency dependence of voltage sensitivity $S=U_{\mathrm{d}} / P$ (here $U_{\mathrm{d}}$ is the detected voltage, $P$ notes the microwave power in a waveguide) is shown in Fig. 2. Essential decrease in the voltage sensitivity from $\approx 800 \mathrm{~V} / \mathrm{W}$ at $f=26 \mathrm{GHz}$ down to $4 \mathrm{~V} / \mathrm{W}$ at $f=178 \mathrm{GHz}$ evidences that the detection mechanism is due to microwave current rectification in the heterojunction. According to this dependence, one should not expect to detect radiation at higher frequencies. However, illumination of the detector with $\mathrm{CO}_{2}$ laser pulses revealed the presence of voltage signal with polarity indicating emission of electrons from $n$-AlGaAs to $n$-GaAs over the potential barrier of the heterojunction.

Temporal profiles of the photoresponse signal and $\mathrm{CO}_{2}$ laser pulse are shown in the inset of Fig. 3. A train of short $\approx 20$ ns laser subpulses was perfectly reproduced by the photosignal in the heterojunction. In addition to fast operation of the detector, the photoresponse trace was found to consist of two components as was observed in the case of Ge, Si and GaAs $l-h$ junctions [3-5]. The fast component that followed the laser pulse can be attributed to the hot carrier photovoltaic effect [2]. Meanwhile, slow relaxation of the signal is determined by thermal component, originating from the crystal lattice heating (Seebeck thermoelectromotive force). As a result, the photoresponse is slightly delayed and has the decay constant of about $5 \mu \mathrm{s}$.

Figure 3 demonstrates voltage-power characteristic of the detector. The photovoltage was measured using the smooth (without subpulses) $\mathrm{CO}_{2}$ laser pulse. 


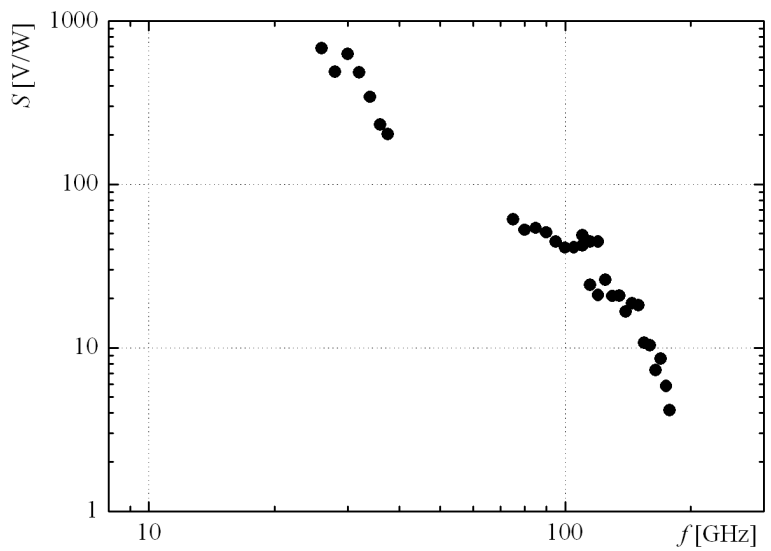

Fig. 2. Frequency dependence of voltage sensitivity of the detector in the microwave region.

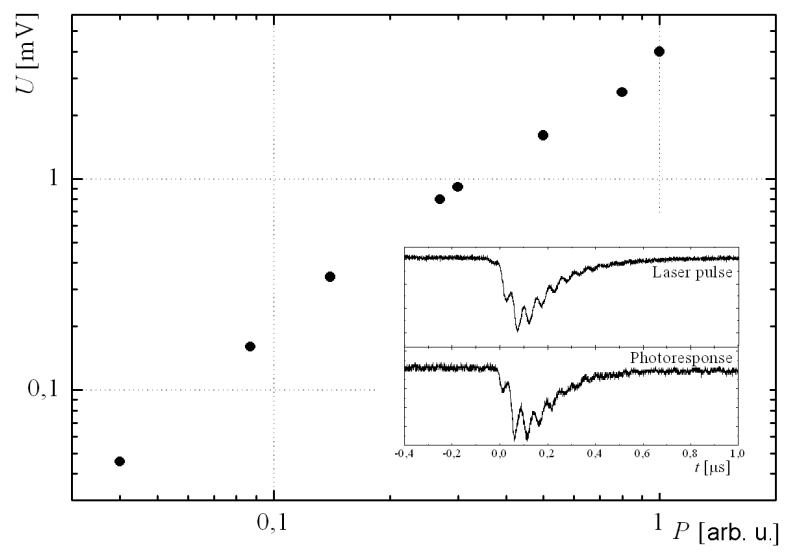

Fig. 3. Voltage-power characteristic of the detector under $\mathrm{CO}_{2}$ laser excitation. The inset shows temporal behavior of the photoresponse signal induced by $\mathrm{CO}_{2}$ laser pulse.

Maximum intensity was about of $100 \mathrm{~kW} \mathrm{~cm}^{-2}$. As in the case of $l-h$ junctions [3-5], the detected signal depends on laser intensity and follows closely a linear law.

One should note that it is possible to decrease the slow component and increase the fast one, if very short pulses of high intensity were used for excitation of the heterojunction. Indeed, the crystal lattice heating is directly proportional to pulse energy absorbed by the sample whereas the hot electron photovoltage increases linearly with pulse intensity [2]. For reasons given above a GaAs/AlGaAs heterojunction is a promising device for subnanosecond IR laser pulse detection.

In conclusion, we have reported an experimental study of photovoltage in GaAs/AlGaAs heterojunction planar detector induced by microwave $(f=26 \div$ $178 \mathrm{GHz})$ and $\mathrm{CO}_{2}$ laser $(\lambda=9.2 \mu \mathrm{m})$ radiation. This device revealed itself as a 
sensitive detector of millimeter waves and as a fast IR sensor operating at room temperature.

\section{Acknowledgments}

This work was partly supported by Lithuanian State Science and Study Foundation under contracts No. V-07051 and No. P-01/2007. The samples were fabricated at Braun Submicron Research Center under contract No. RITA-2003506095. The authors are grateful to Gytis Steikūnas and Angelé Steikūnienè for the sample preparation.

\section{References}

[1] A. Sužiedèlis, S. Ašmontas, J. Požèla, J. Kundrotas, E. Širmulis, J. Gradauskas, A. Kozi; V. Kazlauskaite, T. Anbinderis, Acta Phys. Pol. A 113, 803 (2008).

[2] S. Ašmontas. J. Gradauskas, D. Seliuta, E. Širmulis, A. Michailovas, E. Babrauskas, Mater. Sci. Forum 297-298, 295 (1999).

[3] S. Ašmontas, E. Maldutis, E. Širmulis, Int. J. Optoelectron. 3, 263 (1988).

[4] S. Ašmontas, E. Širmulis, Sov. Phys.-Collection 25, 62 (1985).

[5] S. Ašmontas, J. Gradauskas, D. Seliuta, A. Šilènas, E. Širmulis, in: Proc. Gallium Arsenide Application Symp. GAAS'96, Ed. C. Rumelhard, CNAM, Paris 1996, p. 3B1. 